\title{
A simple device for detecting saccades and judging their intervals
}

\author{
AKIRA OHTANI, YASUO KUCHINOMACHI, and AKIHIRO YAGI \\ Industrial Products Research Institute, Ota-ku, Tokyo, Japan 144
}

\begin{abstract}
A simple device is described with which the occurrence of saccades can be detected and their intervals classified by reference intervals determined by experimental requirements. The device reliably detects saccades of greater than $2 \mathrm{deg}$.
\end{abstract}

Detection of the eye movements and measurement of their time course are useful measurements in various fields of visual research (Ohtani, 1971). Two typical methods of recording eye movements are the eye camera and electro-oculography (EOG). Although an eye camera can measure the exact shift of line of sight, it does not lend itself easily to finding the exact time interval of eye movements. As EOG is an electric analog signal which changes in proportion to the shift of the eye line (Shackel, 1967), it is relatively simple to determine the time course of eye movements, especially in the case of saccadic movements. Furthermore, EOG can be recorded simultaneously with other electrical events (c.g., EEG, ECG, and GSR) on the same magnetic tape for further analysis by a computer.

The simple and inexpensive device described here can detect the occurrence of saccades by analyzing EOG signals. It also can classify the saccades by their intervals. The early stages of the device detect saccadic movements or REM; the later stages classify saccades by reference intervals that are arbitrarily set by the experimenter.

\section{PRINCIPLE OF OPERATION}

A block diagram of the apparatus is shown in Figure 1. The device was designed so that the occurrence of saccades can be detected exactly irrespective of their direction. After horizontal and vertical EOGs are separately processed in pairs of differentiators and full-wave rectifiers for each channel, both EOGs are added with a summing amplifier. The differentiator separates sudden changes of EOG potentials induced by saccades or REM from the slow drift of the potentials accompanied by following movements. The full-wave rectifier converts the biphasic outputs of the differentiator into monophasic so that opposite phasic potentials from the two channels do not cancel at the summing amplifier. The output of the summing

The authors would like to express their gratitude to Gerry Zeitlan (Langley Porter Neuropsychiatric Institute) for his kind inspection of the manuscript,

Akira Ohtani is now at Department Medical Psychology. Hyogo College of Medicine, 1-1 Mukogawacho, Nishinomiya 663, Japan.

The address of Industrial Products Research Institute is 4-21-2 Shimomaruko, Ota-ku, Tokyo, Japan. amplifier is applied to the monostable multivibrator (Mono P), whose output pulses signify the occurrence of the saccadic movements.

If the experiment requires only that the investigator knows the times of the saccadic movements and does not require classifying the intervals of saccades according to critical intervals, the output pulses of Mono P may be used as the signals of saccades.

Classification of saccades' intervals is done in the later stages of the device as follows: An integrator is used for measuring time intervals. The output of Mono $P$ is applied to the base of the transistor that resets the integrator whose output voltage increases linearly in proportion to elapsed time. The output of the integrated is applied to two comparators that compare the time intervals between saccades with reference intervals set by the experimenter. Since the device can classify the intervals of saccades into three categories, two comparators are used. For finer classifications, more comparators may be used.

The output voltage of each comparator changes from logical "l" to logical " 0 " at the moment its input voltage exceeds the reference voltage. The output voltage of each comparator is fed to the corresponding Mono $\mathrm{A}$ and $\mathrm{B}$. These monostable multivibrators are triggered when their input voltage changes from logical " 0 " to logical " 1 ". The outputs of the Mono A and B are fed to AND gates. Mono A is triggered when the saccades' intervals exceed the longer reference interval. AND Gate A, whose two inputs are supplied from the inverted output of Mono $A$, and the noninverted output of Mono B, emits output pulses only when the saccades' interval is between the two reference intervals. AND Gate $B$, whose two inputs are supplies from Mono $P$ and the inverted output of Mono B, emits pulses only when the saccades' interval is smaller than the shorter reference interval.

\section{NOTES ON CIRCUIT DESIGN}

Details of the device, which consists chiefly of monolithic integrated circuits, are shown in Figure 2.

Operational amplifier 709 has no inner circuit for phase compensation. Therefore, a capacitor and a 

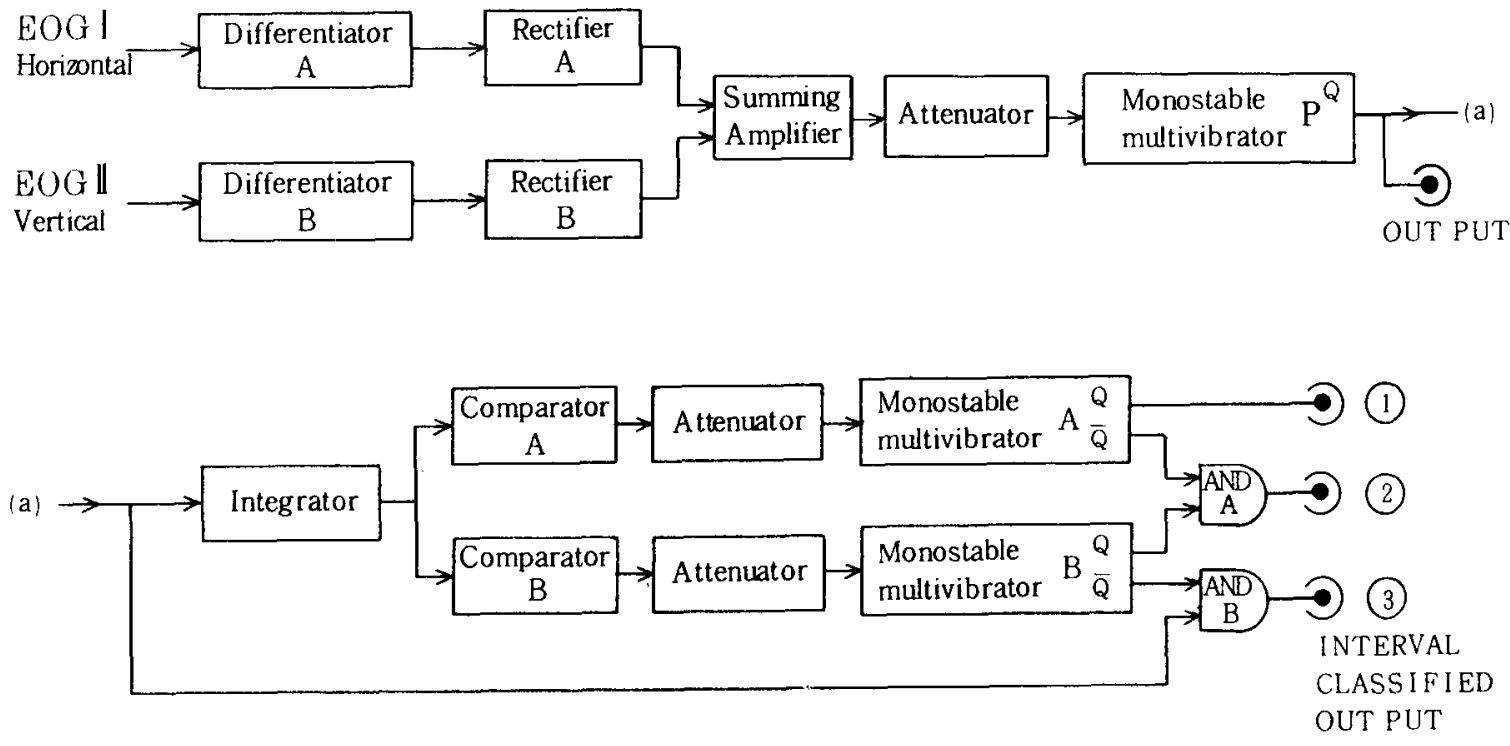

Figure 1. Block diagram of the device.

resistor should be connected in series between Pin 1 and Pin 8, and a capacitor between Pin 5 and Pin 6. An attenuator is placed between the operational amplifier and TTL IC, so that the output voltage of the operational amplifier might be reduced below the recommended input voltage $(5 \mathrm{~V})$ of a TTL IC. The accuracy of resistors and capacitors used in the device are $5 \%$.

\section{Differentiat or}

The time constant to each differentiator is determined by the product of Capacitor $C_{1}$ and Resistor $R_{2}$ shown in Figure 2. It should be set so that the differentiator will pass only the fast change of potentials due to saccades. The time constant of $20 \mathrm{msec}$ had been found to be optimal; this seems reasonable since duration of most saccadic movements is between 20 and $100 \mathrm{msec}$.

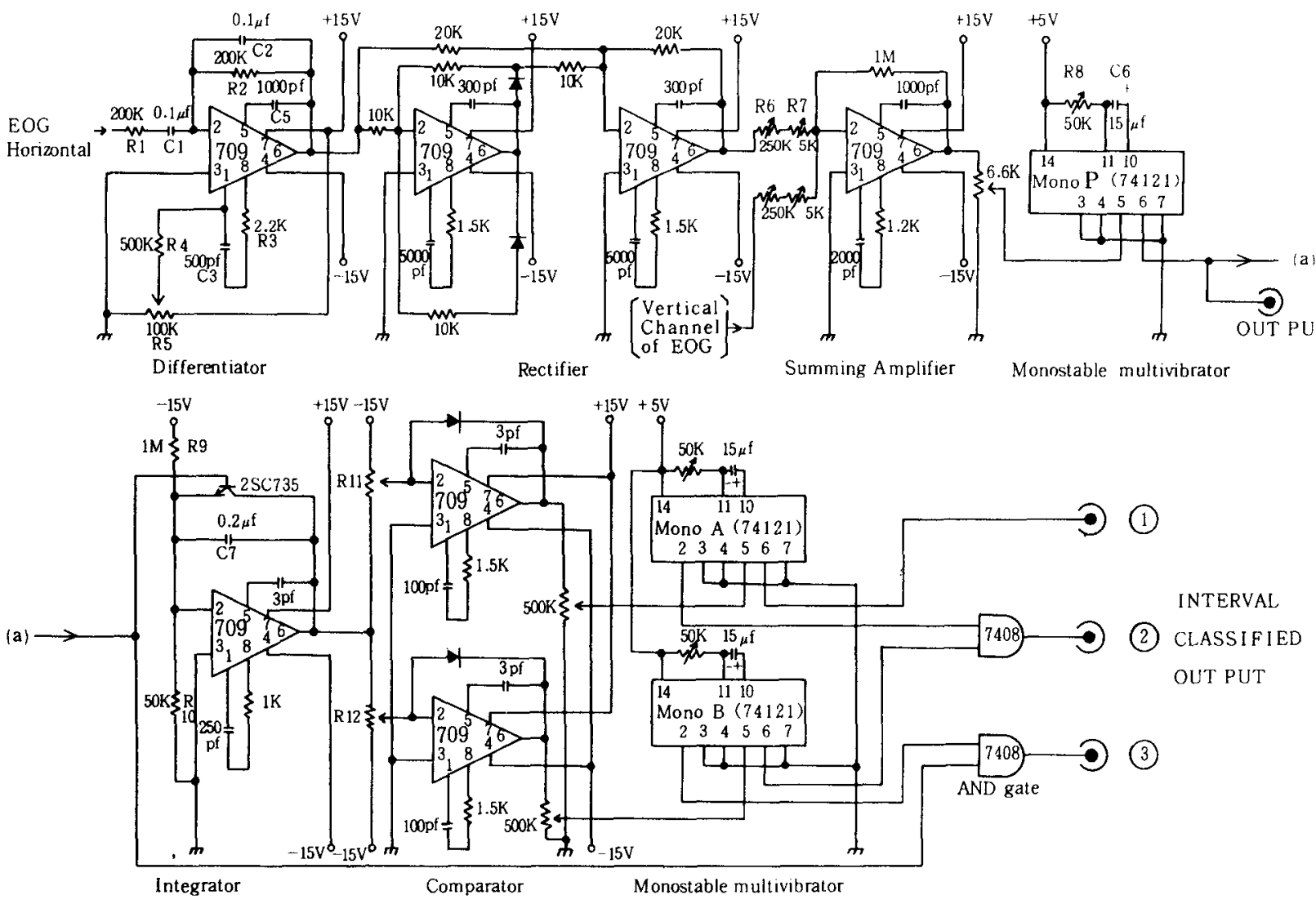

Figure 2. Circuit diagram of the device. ${ }^{3}$ 
Resistor $R_{1}$ and Capacitor $C_{2}$ are attached to avoid mistriggering due to fast noise components riding on the original EOG. The offset voltage at output of the differentiator must be nulled with Potentiometer $\mathrm{R}_{5}$. If any offset voltage remains at the output of the differentiator, the amplitude of the EOG signal with the polarity opposite to the offset voltage is subtracted by the latter. Consequently, the signals that are opposite and smaller than the output offset are never transmitted to the later stages.

\section{Summing Amplifer}

The polarity of the output voltage of the rectifier is always negative. On the other hand, the input polarity of the Mono $P$ must be positive. So the inverting configuration is used in the summing amplifier. The gains of the horizontal and vertical EOG channels are separately controlled by the potentiomenters, $R_{6}$ (rough) and $R_{7}$ (fine). If the gains are too high, the Mono $\mathrm{P}$ is triggered even by noise, and if they are too low, Mono $\mathrm{P}$ is not triggered by small saccades, so they must be carefully adjusted.

\section{Monostable Multivibrator}

The pulse width of Mono $P$, determined by Capacitor $C_{6}$ and Resistor $R_{8}$, is set at $100 \mathrm{msec}$. This pulse width was chosen to avoid mistriggering by damping oscillations which occur immediately after termination of very large eye movements lasting nearly $100 \mathrm{msec}$. As it is rare to find a shorter saccadic interval than $100 \mathrm{msec}$, the 100 -msec pulse width does not noticeably impair the performance of the device.

\section{Integrator}

The time constant and the constant input voltage supplied to the inverted input of the integrator are selected so that its output voltage might not reach the saturation level within the longest reference interval. The time constant is determined by Resistors $R_{9}$ and $R_{10}$ and Capacitor $\mathrm{C}_{7}$. The voltage for the constant input is obtained by means of dividing the negative power supply of $-15 \mathrm{~V}$ with these resistors.

The transistor (Toshiba 2SC735) used in the switching circuit influences the performance of the device. In order to achieve negligible reset time, it must have the following characteristics: (a) high gain $\left(\mathrm{h}_{\mathrm{FE}}\right)$, (b) large $\mathrm{I}_{\mathrm{c}}$ and (c) small leakage current.

\section{Comparator}

The reference voltage is set by adjusting Potentiometer $R_{11}$ or $R_{12}$ so that the output of the comparator changes for logical " 1 " to logical " 0 " immediately after the corresponding reference interval passed.

\section{TEST OF THE DEVICE}

We tested the device in order to determine the smallest saccades that could be detected. The subjects were three males. The stimuli, located $1 \mathrm{~m}$ in front of the subjects, were two dots whose visual angles were changed from 10 to $1 \mathrm{deg}$. The inclination of dots in the horizontal plane was changed by steps of $22.5 \mathrm{deg}$. Subjects were required to move their eyes alternatively between the two dots. A pair of $\mathrm{Ag} \cdot \mathrm{AgC} 1$ electrodes (Beckman) was placed at the outer canthi of the two eyes to measure horizontal movements. Another pair was placed above and below the right eye for vertical movements. EOGs were amplified by high gain $\mathrm{dc}$ amplifiers (Nihonkoden, RUD-5) and recorded on magnetic tape by a Sony DFM-45 FM tape recorder for subsequent analysis. Horizontal and vertical EOG signals to the differentiator were set to $100 \mathrm{mV} / \mathrm{deg}$ for corresponding eye movements.

Where visual angles change from 10 to $2 \mathrm{deg}$, Mono $P$ emits pulses in response to all the saccades regardless of the inclination of the dots. When the gain of the summing amplifier is increased so that Mono $\mathrm{P}$ is triggered even by 1 -deg saccades, Mono $P$ is spuriously triggered hy EEG alpha waves or by electric noise. This occurs becalise the EOG change due to 1-deg saccades is nearly the same as that due to alpha waves. The optimal anlustment of the summing amplifier gain is such that Mono P is a riggered by visual angle changes of $2 \mathrm{deg}$ or greater.

We have another electric analog signal similar to the EOG signal by the photocell method of measuring a reflected light beam off the corneo-scleral junction. Analysis of the signal taken from the photocell is expected to bring similar results by the authors' device.

\section{REFERENCES}

Ohtani, A. An analysis of eye movements during a visual task Ergonomics, 1971, 14, 167-174.

Shackel, B. Eye movement recording by electro-oculography. In P. H. Venables, \& I. Martin (Eds.), A manual of psychophysiological methods. Amsterdam; North-Holland, 1967.

\section{NOTE}

1. The diagram for the vertical is omitted in Figure 2 since the circuit for the vertical channel preceding the summing amplifier duplicates the horizontal circuit.

(Received for publications March 8, 1974, revision accepted September 20, 1974.) 